\title{
A Search for Wolf-Rayet Stars in the Small Magellanic Cloud
}

\author{
Philip Massey ${ }^{1}$ \\ Lowell Observatory \\ 1400 W. Mars Hill Road, Flagstaff, AZ 86001 \\ massey@lowell.edu \\ and \\ Alaine S. Duffy ${ }^{1,2}$ \\ Gettysburg College \\ Box 756, Gettysburg, PA 17325 \\ s432014@gettysburg. edu
}

Received —

\footnotetext{
${ }^{1}$ Visiting Astronomer, Cerro Tololo Inter-American Observatory, National Optical Astronomy Observatory (NOAO), which is operated by the Association of Universities for Research in Astronomy, Inc. (AURA) under cooperative agreement with the National Science Foundation (NSF).

${ }^{2}$ Participant in the NSF's Research Experiences for Undergraduates program, Summer 2000.
} 


\begin{abstract}
We report on a comprehensive search for Wolf-Rayet (W-R) stars in the SMC using interference-filter imaging. Photometry of over 1.6 million stellar images on multiple, overlapping fields covering 9.6 square-degrees found the previously known W-Rs at very high significance levels, two known Of-type stars, plus additional candidates which we examined with slit spectroscopy. We discovered two new Wolf-Rayet stars, both of type "WN3+abs", bringing the total number in the SMC to 11. We discuss their spectra, as well as reclassifying the previously known ones with our new data. Our survey also revealed 4 newly found Of-type stars, including one of O5e If + type, one of the earliest-type stars known in the SMC. Another newly identified Of star is AV 398 (O8 If), a star often used in extinction studies under the assumption that it is of early B type. We recover S18 (AV154), a B[e] star whose spectrum currently lacks He II $\lambda 4686$ emission, but which must have had strong emission a year earlier; we compare this star to $\mathrm{S}$ Dor, suggesting it is indeed a luminous blue variable (LBV). We also find a previously unknown symbiotic star whose spectrum is nearly identical to the Galactic symbiotic AG Dra. More important, perhaps, than any of these discoveries is the demonstration that there is not a significant number of W-Rs waiting to be discovered in the SMC. The number of W-Rs is a factor of 3 times lower in the SMC (per unit luminosity) than in the LMC. This strongly suggests in that at the low metallicity which characterizes the SMC that only the most massive stars can evolve to W-R type.
\end{abstract}

Subject headings: Magellanic Clouds — stars: early-type — stars: evolution — stars: emission-line, Be — stars: Wolf-Rayet 


\section{Introduction}

For many years, our knowledge of the Wolf-Rayet (W-R) content of the SMC was thought to be complete, with a total of eight discovered. Of these, four had been found by general studies of the SMC's blue stellar population (see Breysacher \& Westerlund 1978), with an additional four found by an objective prism survey targeted specifically at detecting W-Rs by using an interference filter to reduce sky background and crowding (Azzopardi \& Breysacher 1979). There matters stood until the discovery of Morgan, Vassiliadis, \& Dopita (1991) of a ninth W-R. This star was well within the boundary surveyed by Azzopardi \& Breysacher (1979), but a few tenths of a magnitude fainter than any of the others.

Wolf-Rayet stars are the evolved, He-burning descendents of the most massive stars, and their strong emission lines allow them to serve as important tracers of the young stellar population in other galaxies (Massey 1985). The number of W-R stars in the SMC is of interest, as it appears to be disproportionately low compared to that in the LMC, where roughly 130 are known (Breysacher, Azzopardi, \& Testor 1999). The (visual) luminosity of the SMC is only about a factor of 3.6 less than that of the LMC (Table 2 of van den Bergh 2000a), and, all other things being equal, we would thus expect $~ 35 \mathrm{~W}$-Rs in the SMC. Thus the presence of only $9 \mathrm{~W}$-Rs suggests an under-abundance by a factor of $\sim 3.5$.

This scarcity, if real, is telling us something interesting either about the progenitor massive star population in the SMC, or about how its massive stars evolve in the SMC. Let us examine these two possibilities in turn.

Since the visual luminosity is dominated by an older population, it could simply be that the massive stars from which W-R evolve are scarcer in the SMC due to a steeper initial mass function (IMF) and/or a lower current star formation rate (SFR). What is the available evidence? Massey et al. (1995) find that the IMF for massive stars appears to be indistinguishable between the SMC and the LMC (and with the Milky Way for that 
matter). In that case, a lack of massive progenitors would require a lower SFR in the SMC. Could the current SFR be 4 times lower in the SMC than in the LMC? Let us compare the number of OB associations in the two Clouds. Hodge (1985) catalogs 70 regions in the SMC, compared with the 120 found by Lucke \& Hodge (1970) in the LMC. (Care was taken to use the same selection criteria in the two galaxies, and indeed the size distributions are indistinguishable.) This would suggest that the SMC has a SFR a factor of 2 higher per unit luminosity than does the LMC. A better measure of the SFR would be the integrated $\mathrm{H} \alpha$ luminosity. However, what is available is not quite this, but rather the total of all the $\mathrm{H} \alpha$ emission from the discrete H II regions. Kennicutt (1991) finds that these require an

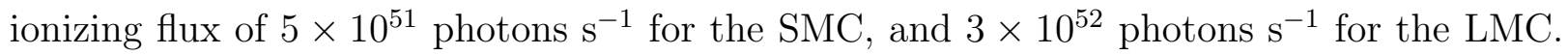
The factor of 6 difference would suggest that the SFR is 1.6 times lower per unit luminosity in the SMC. These totals do not include losses from density-bounded H II regions, which should result in diffuse emission; such a correction may or may not be the same for the two Clouds. In any event, these data suggest that a lack of massive stars is not the primary cause for the factor of 3-4 under-abundance of W-Rs in the SMC compared to the LMC.

On the other hand, we have good reason to suspect that the low metallicity of the SMC will have a significant effect on the evolution of massive stars, as there are smaller mass-loss rates at a given luminosity. In the "Conti scenario" (Conti 1976; Maeder \& Conti 1994) Wolf-Rayet stars are produced as a massive star loses mass via stellar winds; during the He-burning stage the outer layers have been sufficiently stripped to reveal the H-burning products at the surface and the star is spectroscopically identified as a WN-type Wolf-Rayet. Further mass-loss leads to a WC-type, with the products of He-burning (C, O) revealed in the spectrum. The strong, broad emission lines are the result of a highly extended and rapidly expanding stellar atmosphere; e.g., optical depth unity occurs at a point in the stellar wind. We would thus expect that stars that evolve to W-Rs in the SMC would have to be of higher luminosity (and mass) than those in galaxies with higher 
metallicity. (The oxygen abundance is a factor of 1.7 lower in the SMC than in the LMC, according to Russell \& Dopita 1990). Indeed, the high proportion of WN-type Wolf-Rayets to those of WC-type in the SMC (8:1) is consistent with the hypothesis that WCs are harder to produce at low metallicities due to smaller mass-loss rates, and is in accord with the SMC's metallicity compared to other Local Group galaxies (Massey \& Johnson 1998). Recent studies of coeval clusters and associations suggest that "cut-off" mass for becoming a W-R star is about $70 \mathcal{M}_{\odot}$ in the SMC, while stars with initial masses greater than $30 \mathcal{M}_{\odot}$ become W-Rs in the LMC (Massey, Waterhouse, \& DeGioia-Eastwood 2000b); in the Milky Way the limit may be as low as $18 \mathcal{M}_{\odot}$ (Massey, DeGioia-Eastwood, \& Waterhouse 2000a).

However, the discovery of the ninth SMC W-R by Morgan et al. (1991) raises the question of the completness of previous surveys. We were naturally curious if a significant additional population of $\mathrm{W}$-Rs remained to be found in the SMC. We have had good success in searching for W-Rs in nearby galaxies using a set of interference filters optimized to detect W-Rs (e.g., Armandroff \& Massey 1985, Massey, Armandroff, \& Conti 1986; Massey \& Johnson 1998). Accordingly we decided to survey the SMC using the CTIO/Michigan Schmidt with these filters during a gap in our main observing program last year. Follow-up spectroscopy this year identified 8 hitherto unknown interesting objects in the SMC, including 4 "Of" stars, a luminous blue variable (LBV) candidate, a symbiotic star, and two newly found Wolf-Rayet stars. We describe our survey, these newly found objects, and the implications here. 


\section{The Search for New Wolf-Rayet Stars}

\subsection{The Survey}

Images were taken with the CTIO Curtis Schmidt telescope during (UT) 1999 October 23-28. The field-of-view was $1.3^{\circ} \times 1.3^{\circ}$ using the Tektronix 2048 detector; the scale was 2.3 arcsec pixel $^{-1}$. Outside the central $1000 \times 1000$ pixel region our 2 -inch filters caused vignetting, with the amount of light decreasing to $30-40 \%$ on the sides, and to $5-15 \%$ in the extreme corners. We covered a 9.6 square-degree area centered on the SMC with 12 (very) overlapping fields to make the effects of this vignetting minor. The region surveyed is shown in Fig. 1, where we have also indicate the area covered by Azzopardi \& Breysacher (1979).

Our interference filter set is described in detail by Massey \& Johnson (1998): the $W C$ filter is centered on C III $\lambda 4650$, the $W N$ filter is centered on He II $\lambda 4686$, and the continuum $C T$ filter is centered at $4750 \AA$. The band-passes are approximately $50 \AA$ wide. The effective wavelengths of these filters used in the fast $\mathrm{f} / 3.5$ beam of the Schmidt were

close to these nominal values (4646 $\AA$ for the $W C$ filter and $4685 \AA$ for the $W N$ filter).

For each of the 12 fields, we obtained 3 consecutive $600 \mathrm{sec}$ exposures in each of the 3 filters (i.e., 9 per field). The data were biased and trimmed in the usual manner, with bright twilight exposures serving as our flat-fields. Stars brighter than $V \sim 11.5$ were saturated.

Owing to the under-sampling, we performed only aperture photometry on the images. Nevertheless, analyzing the frames was a daunting task, with over 1.6 million stellar images measured. Each image was treated separately, with the photometry averaged for the 3 exposures through each filter at the end. A 2.5 pixel (5.8 arcsec) radius aperture was used throughout. Observations of several galactic W-R stars were used to set the instrumental zero-point of the $C T$ magnitudes to that of $v$ (an emission-free equivalent of $V$ introduced by 
Smith 1968b) with the assumption (borne out by the results) that there were minimal color effects. Photometry from field to field was remarkably consistent, with typical differences $\sim 0.01$ mag for bright stars from night to night.

In order to identify $\mathrm{W}-\mathrm{R}$ candidates we obtained the magnitude differences $W C-C T$ and $W N-C T$ for all the objects in a field. Stars significantly brighter in one of the emission-line filters than in the continuum filter were judged to be likely candidates. As in our earlier work, "significantly brighter" was determined by using the actual statistical uncertainties of the photometry; a magnitude difference had to be at least five times this value (i.e., $5 \sigma$ ) to be considered a viable candidate. In addition, we ignored stars which appeared only 0.05 mag brighter in one of the on-line filters. We expect a difference of 0.1 mag to correspond roughly to an emission-line equivalent width of -10 ÃA, about the limit of Wolf-Rayet stars (Massey \& Johnson 1998 and discussion therein).

The results of our analysis were encouraging. With the exception of HD 5980 (which was saturated), all of the known SMC W-R stars were found repeatedly on every field they appeared, typically with significance levels of $30 \sigma$ or more. For instance, the faintest previously known W-R star, the one discovered by Morgan et al. (1991), with $v \sim 15.3$, was identified as a W-R "candidate" on all 3 fields in which it appeared, with a $W N-C T$ difference of $-0.42 \mathrm{mag}$ at a significance level of $11 \sigma, 16 \sigma$, and $12 \sigma$. The stronger-lined, brighter W-R star AB4 $(v=13.2)$ was found with a $W N-C T$ difference of $-0.80 \mathrm{mag}$, at a significance level of $78 \sigma$ and $87 \sigma$ on the two overlapping frames on which it appeared. Although the W-R star HD 5980 itself was saturated on our frames, the nearby strong-lined O7 If + star Sk 80 was detected (due to He II $\lambda 4686$ emission) on all four overlapping frames that included that region, with a $W N-C T=-0.11 \mathrm{mag}$ at a significance level of $23-29 \sigma$. Thus we had some confidence that our detection technique would find any remaining W-R stars even if they were significantly fainter and/or weaker-lined than the known ones. 


\subsection{Followup Spectroscopy}

Spectra of all the good candidates were obtained on the night of (UT) 2000 Oct 10 with the CTIO 1.5-m CCD spectrometer. A 527 line $\mathrm{mm}^{-1}$ grating (No. 16) was used in first order with no blocking filter to provide spectral coverage 3990-5950 $\AA$ with a spectral resolution of $4.2 \AA\left(1.5 \AA\right.$ pixel $\left.^{-1}\right)$. A 3 arcsec slit was used in order to provide reasonable spectrophotometry. The known SMC W-Rs (with the exception of HD 5980) were also observed, as well as the O7 If star Sk 80. On subsequent nights (2000 Oct 11-14) additional data were taken on the most interesting stars (including HD 5980) at higher resolution using the 831 line $\mathrm{mm}^{-1}$ (No. 47) in second order with a $\mathrm{CuSO}_{4}$ blocking filter. This set-up provided a wavelength coverage of 4090-4750 A, with $1.4 \AA$ resolution $\left(0.5 \AA\right.$ pixel $\left.^{-1}\right)$ with a 1 arcsec slit. Our reductions followed the usual procedures, with the flat-fielding accomplished by exposures of the "punto blanco" (3-4 hrs in the case of the higher dispersion grating). For the spectral extractions, we used the optimized extraction routines within IRAF, with care being take to select clean sky on other side of the object. In the case of nebulosity we performed several extractions in an effort to minimize the under- or over-subtraction of nebular emission.

Not unexpectedly, most of our candidates turned out to be bogus for one reason or another, usually related to the poor spatial resolution of the Schmidt images; e.g., two stars of very different colors would be unresolved on our frames. We also expect spurious detections (particularly those found on a single frame) simply due to the number of $5 \sigma$ outliers expected given the large number of stars photometered. Many of our W-R candidates proved to be K-type dwarfs, but which were detected at high significance level due to the strong, broad $\mathrm{MgH} \lambda 4780$ feature which depressed the $C T$ magnitude. A total of $\sim 25$ unknown sources were examined, of which a third proved of interest.

We discuss our more successful discoveries in the following section. 


\section{Results}

\subsection{The Wolf-Rayet Content of the Small Magellanic Cloud}

Two of our high-significance candidates proved to be Wolf-Rayet stars of WN3 type. We list their properties in Table 1, along with those of the other SMC W-Rs ${ }^{3}$. In addition to the spectrophotometry from our spectra, we also have Curtis Schmidt $B V R$ images of our fields obtained on 1999 Jan 8 as part of our search for red supergiants in the SMC; we have used these data to provide $V$ and $B-V$ photometry for our stars. Although "line-free" $v$ and $b-v$ values for the W-R stars would be preferable (Smith 1968b), we have used our spectrophotometry to substantiate that the correction is, in general, minor for these relatively weak-lined W-Rs, and we use the broad-band colors to correct for reddening until we can obtain better calibrated spectrophotometry. (The exception is the newly found W-R SMC-WR10, for which nebulosity significantly the $B-V$ colors, and hence we have used the spectrophotometry.) We adopt a single $(B-V)_{o}=-0.32$ for all the W-R subclasses (see Fig. 4 of Pyper 1966). A value of $A_{V}=0.1$ corresponds to a realistic minimum reddening, and $A_{V}=0.3$ is typical for early-type stars in the SMC (Massey et al. 1995); the average $A_{V}$ of the $\mathrm{W}$-Rs is about 0.7 mag, suggesting we may be overestimating the reddening correction slightly by this method. A true distance modulus of 18.9 (van den Bergh 2000b) was adopted in computing $M_{V}$.

The SMC W-Rs were throughly discussed by Conti, Massey, \& Garmany (1989), who used SIT-Vidicon spectrophotometry along with moderate resolution photographic spectra

\footnotetext{
${ }^{3}$ We refer to these two newly found W-Rs as SMC-WR10 and SMC-WR11, consistent with the IAU nomenclature recommendations, and suggest that the previously known W-Rs (often referred to as "AB1" through "AB8" and "Morgan's star") be known as SMC-WR1 through WR9.
} 
to provide descriptions of each of the 8 SMC W-R stars known then. (See also Moffat 1988.) For consistency, we reobserved all of the SMC W-Rs along with our newly found ones, and reclassified them ab initio. We are in good agreement with Conti et al. (1989) and Moffat (1988) except as noted below. Since these data have somewhat higher resolution and better signal-to-noise we briefly comment on each of the SMC W-Rs here. We illustrate their spectra in Figs. 2 and 3.

All but SMC-WR4 show absorption lines in their spectra, including our two newly identified W-Rs. Conti et al. (1989) emphasize that this could be due to these being binaries or due to their having thin stellar winds allowing photometric absorption. Moffat (1988) suggests that five of these (WR3, WR5, WR6, WR7, and WR8) are double-lined spectroscopic binaries. Of these, orbits have been determined for three of these: WR6 (Hutchings et al. 1984) WR7 (Moffat 1988; Niemela \& Morrell 1999), and WR8 (Moffat, Niemela, \& Marraco 1990), with the issue for WR5 (HD 5980) very complicated (Koenigsberger et al. 2000 and references therein). A radial velocity study of the SMC W-Rs is currently underway, and for now we will refer to the ones without established orbits as "WR+abs" and separately describe the absorption spectra.

SMC-WR1: At $V=15.1$ this was the faintest of the original eight SMC W-Rs. The star has strong He II $\lambda 4686$ and N V $\lambda \lambda 4603,19$ emission, but no N IV $\lambda 4058$ or N III $\lambda \lambda 4634,42$ emission. (Conti et al. 1989 note the presence of N IV $\lambda 3480$.) He II emission at $\lambda 4859$ and $\lambda 5411$ are visible, and the W-R star is readily classified as "WN3". A faint absorption spectrum was reported by Moffat (1988), described as "O4:". On our spectra we see only He II absorption lines $(\lambda \lambda 4200,4542)$ plus the Balmer lines, and we call the absorption component O3-4.

SMC-WR2: This star has narrow emission at He II $\lambda$ 4686, N IV $\lambda 4058$; $\mathrm{N}$ III $\lambda \lambda 4634,42$ is weakly present in emission, and we classify the star as WN4.5. In 
addition emission at He II $\lambda 4859$ and $\lambda 5411$ is present. C IV $\lambda 5808$ is clearly present in our spectrum. Its strength is too weak $(20 \times)$ to consider this a transitional WN/C type (see Fig. 5 of Conti \& Massey 1989) by Galactic or LMC standards, although its singular presence in this star in the metal-poor SMC suggests that this may actually be "on its way" from WN to WC type. Conti et al. (1989) describe the absorption spectrum as being of "early O type", in agreement with our spectrum, which we tentatively describe as O5: Conti et al. (1989) note this star shows no discernible radial velocity variations.

SMC-WR3: At first glance, this star is readily classified as "WN3+abs": there very strong He II $\lambda 4686$ and N V $\lambda \lambda 4603,19$ emission, as well as the He II $\lambda 4859$ and $\lambda 5411$ in emission. We were puzzled by the very faint, but unarguable presence of N III $\lambda \lambda 4634,42$ emission in our spectrum, given the total absence of N IV $\lambda 4058$. Such a possibility is not allowed for in the classification scheme. Admittedly our signal-to-noise is higher than that used to develop the classification criteria. The faint absorption spectrum is impossible to classify given emission at He II $\lambda 4542$; however, He I $\lambda 4471$ is clearly present. Moffat (1988) argues that this star is an SB2 with a period longer than a week, but a true orbit has never been published for this star. We speculate that the very faint N III emission may belong to the O-type star if the spectrum is truly composite.

SMC-WR4: shows moderately strong N IV $\lambda 4058$ comparable to N III $\lambda 4634,42$ emission, with weak or absent N V $\lambda 4603,19$ emission. We do see signs of weak N V absorption, although no other absorption lines are seen in the spectrum. Thus by the standard classification scheme (Smith 1968a) we would call this star a WN6p, with the "peculiar" designation a reminder of the N V absorption. Conti et al (1989) did not see any N III $\lambda \lambda 4634,42$ and thus called the star a "WN4.5" However, based upon photographic spectra obtained at about the same time, Moffat (1988) had called the star a WN6, so we believe this is not a real spectral change. There are no other absorption lines visible. 
SMC-WR5: HD 5980 underwent an "LBV-like" outburst (Barba et al. 1995), after which its spectrum was no longer "OB+WN3". Niemela, Barba, \& Morrell (1999) describe its spectrum as having evolved to a very late WN type, which they describe as "WN11". We have only a higher resolution spectrum which does not include coverage of the N IV $\lambda 4058$ region, but both $\mathrm{N}$ III and $\mathrm{N}$ V are present, and we might call this a WN5 without additional information. Strong lines of He II are present. Our spectrum does show an earlier type than the latest (1997) spectrum illustrated by Niemela et al. (1999). Doubtless further monitoring will determine if the star is evolving back towards earlier type or not. The photometry we give is from 1999 Jan; we lack photometry of this star in its present state, but an intensive monitoring campaign by others (i.e., Niemela et al. 1999) is currently underway.

SMC-WR6: Our spectra show He II $\lambda 4686$ and modest N V $\lambda \lambda 4603,19$ emission, without either N IV or N III, leading to the "WN3" classification. A strong absorption-line spectrum is present, which we classify as O7 type. Moffat (1988) refers to the companion as O6.5, consistent with our designation.

SMC-WR7: Conti et al. (1989) refer to SMC-WR7 simply as "WN+abs"; our high signal-to-noise spectrum shows no lines of nitrogen, and we would call this a WN2+abs using the "extended" criteria of van der Hucht et al. (1981) that WN2 have weak or absent N V. We do see He II $\lambda \lambda 4542,5400$, consistent with the admonishment that WN2 stars have "strong He II". A strong absorption spectrum is present, which we classify as O6 type, similar to the "O7:" description of Moffat (1988).

SMC-WR8: The only WC-type Wolf-Rayet known in the SMC, the optical spectrum is dominated by C III $\lambda 4650$ and C IV $\lambda 5808$. No C III $\lambda 5696$ is seen, but strong O V $\lambda 5592$ is present, and would lead to a WC4 classification. Our spectrum does not go far enough into the blue to detect the strong O VI $\lambda 3820$ emission which led to this being considered 
an WO4 star (Moffat, Breysacher, \& Seggewiss 1985), but OVI $\lambda 5920$ is readily visible in the yellow-red, as expected (Crowther, De Marco, \& Barlow 1998). The absorption component was described by Moffat et al. (1985) as O4-type, in agreement with the "O4 V" classification by Massey et al. (2000b).

We next consider the spectrum of the Wolf-Rayet star found by Morgan et al. (1991) and our two newly found W-Rs. The spectra are shown in Fig. 3.

SMC-WR9: Morgan et al. (1991) classify SMC-WR9 as "WN2.5+abs"; our spectrum of this star shows modest $\mathrm{N} \mathrm{V}$ emission in good accord with their spectrum. We classify the star as WN3+abs, a difference due more to our interpretation of the spectral subclasses than with any disagreement with the spectral description by Morgan et al. We classify the absorption component as O3-4, based on strong He II absorption and an apparent lack of He I $\lambda 4471$. This is slightly earlier than the "O5:" designation of Morgan et al.

SMC-WR10: This star is embedded in very strong nebulosity, the NW knot of e12 (NGC 249), illustrated on plate 101V of Hodge \& Wright (1977). This doubtless contributed to the difficulty of finding it via objective prism techniques. Our spectrum shows He II $\lambda 4686$ with a similar full-width-at-half-maximum (fwhm) and equivalent width to the other W-R WNs. N V $\lambda \lambda 4603,19$ emission is strongly present, as is He II emission at $\lambda 4859$ and $\lambda 5411$. There is no sign of N IV $\lambda 4058$ or N III $\lambda 4634,42$, making it of WN3 type. Our higher resolution spectrum shows absorption at He II $\lambda \lambda 4200,4542$, as well as $\mathrm{H} \gamma$; we classify the absorption component as $\mathrm{O} 3$.

SMC-WR11: Our other newly found W-R star is fairly weak lined, and is the only star with appreciable reddening, but is otherwise unremarkable. The star is not a member of any OB association or H II region, nor is it particularly crowded; only its faintness and weak lines can account for its lack of previous attention. The presence of strong N V $\lambda \lambda 4609,20$ emission and the lack of either N IV $\lambda 4058$ or N III $\lambda \lambda 4632,42$ argues again 
for the WN3 subclass; Balmer absorption and He II $\lambda 4200,4542$ is likewise revealed on the high-resolution spectrum, leading us to classify the absorption as O3-4 due to the apparent lack of He I $\lambda 4471$.

We provide finding charts for all 11 of the SMC W-Rs in Fig. 4.

\subsection{Other Finds}

We list in Table 2 the other stars we found of interest, and discuss these here. Finding charts are given in Fig. 5.

\subsubsection{Of-type Stars}

Wolf-Rayet stars are not the only early-type stars with He II $\lambda 4686$ emission, of course. The most luminous O-type stars (e.g, luminosity class "I") also show He II $\lambda 4686$ emission, as well as N III $\lambda \lambda 4634,42$ emission. We aimed our selection to be sensitive to the weakest-lined W-Rs, and it is therefore not surprising that some strong-lined Of-type stars were detected. Two of these were previously known: AV 220, of type O7 If (Garmany, Conti, \& Massey 1987), and Sk 80, an extreme O7 If+ star (Walborn \& Fitzpatrick 1990). However, our survey also uncovered four previously unknown Of stars, and we list these

in Table 2. One of these is of type O5e If, making it one of the earliest-type supergiants known in the SMC. We have adopted $(B-V)_{o}=-0.30$ in determining the correction for interstellar extinction.

Interestingly three of the four newly found Of stars do not appear in the Azzopardi \& Vigneau (1982) catalog of SMC members. At $V \sim 13.4-14.0$ these are considerably brighter than the $V \sim 15$ plate limit of the Azzopardi \& Vigneau $(1975,1982)$ survey; all 
three are within the survey region (two on their chart 5, and one on their chart 4). All three are located in the bar, arguing that perhaps crowding may have made their detection difficult.

Here we discuss the four stars in turn.

MA93-344: This Of star was cataloged as an $\mathrm{H} \alpha$ emission-line star by Meyssonnier \& Azzopardi (1993). We show the spectrum in Fig. 6. Our efforts to obtain a suitable high resolution spectrum of this star failed due to clouds on our last observing night; the lower resolution spectrum we show in Fig. 6 reveals an O9-type spectrum with broad He II $\lambda 4686$ emission with an absorption feature superposed. There is no trace of N III $\lambda 4634,42$ emission. Were this star a Galactic star we would be tempted to cite a binary explanation, with the companion possibly of W-R type. Si IV $\lambda 4089$ is strong, supporting the interpretation that the O star is of luminosity class "I". Keeping in mind that we do not fully understand the behavior of "Of" features in the low metallicty environment of the SMC, we stay with the minimalist classification O9 If, but do not exclude the possibility this star is a binary with a twelfth W-R companion.

Anon-1: This star has very strong emission for an Of-type star. In Fig. 7 we compare it to the "extreme" Of star Sk 80. In addition, narrow Balmer emission is seen on top of absorption. We obtained several spectra of this star, and it does not appear to be a binary, in that the spectral morphology did not change over several nights. The very narrow He II $\lambda 4686$ line $(6 \AA \mathrm{fwhm})$ argues that this is an Of rather than a composite $\mathrm{W}-\mathrm{R}$ type. The $-5 \AA$ equivalent width is strong for a Of star, but not unprecedented; the Galactic O8 If star HD 152408 does have comparable He II $\lambda$ line strength (Conti \& Leep 1974). Although not unique, the line-strength is unusual. The star is located in OB association 35 (Hodge 1985), along with S18, the LBV we will discuss in the following section.

Anon2: The spectrum is that of an O7 If. N III $\lambda \lambda 4634,42$ is anomalously strong, and 
He II $\lambda 4686$ shows absorption superposed on emission.

AV398: The fourth newly found Of star, AV 398, has been classified as "O9Ia:" from an IUE spectrum by Neubig \& Bruhweiler (1997), who quote the optical spectral type as "B2" based upon the objective prism classification of Azzopardi \& Vigneau (1975). Our

spectrum clearly reveals a normal O8 If star (Fig. 6). Interestingly this star has long been used as a "typical bar line of sight" star for studies involving the extinction law of the SMC (most recently Zubko 1999). Such studies eschew emission-line stars (see discussion in Rodrigues et al. 1997); apparently the objective prism classification was taken literally.

\subsubsection{Two Other Emission-line Stars}

Our survey detected the star S18=AV 154, a B[e] star that has many LBV-like characteristics (Morris et al. 1996). S18 was detected on three overlapping fields, observed during consecutive nights, and during this time the $C T$ magnitude brighten from 13.55 to 13.74. Our spectrum was taken a year later. We were initially puzzled that we detected the star at all given the 2000 Oct spectrum, as there is no He II $\lambda 4686$ present. (The star turned up as a $95 \sigma$ candidate!) However, the spectrum shown by Shore, Sanduleak, \& Allen (1987) clearly does show strong He II $\lambda 4686$. Its absence during 2000 Oct is not without precedent, however, as Zickgraf et al. (1989) also reported the line as having been missing in a 1987 spectrogram. This star is discussed extensively by Morris et al. (1996), who emphasize the need for further monitoring. Here we draw attention to its spectral similarity to S Dor, the archetypical LBV, during its 1996 Oct "low" state (Massey 2000). We compare the two in Fig. 8. Given the photometric variability, high luminosity, spectral changes, and its spectral similarity spectrally to S Dor, one would be hard-pressed not to consider this an LBV.

The star is located in OB association 35 (Hodge 1985), the same association which 
our newly discovered O5e If star is found. Our study of the environments of LBVs in the Magellanic Clouds and Milky Way suggest that the LBVs which are members coeval regions nearly are always found with extremely massive stars. A detailed study of h35 is doubtless warranted.

The last star of interest was included in the list of $\mathrm{H} \alpha$ emission-line stars given by Meyssonnier \& Azzopardi (1993), their number 1748. Nothing else was previously known about the star. Its spectrum, shown in Fig 9, was clearly of a symbiotic star. It does not

appear in the recent catalog of symbiotic stars by Belczynski et al. (2000), and appears to be only the seventh such object known in the SMC. Howard Bond and Tony Keyes kindly pointed out its similarity to that of the Galactic symbiotic star AG Dra, illustrated in Fig A.14 of Kenyon (1986). With $V=16.61$, we would estimate an absolute magnitude of -2.6 , not inconsistent with the typical $M_{V}=-2$ quoted by Kenyon (1986).

\section{Discussion}

Our survey discovered two previously unknown Wolf-Rayet stars, bringing the total number in the SMC to 11. Although we cannot preclude a Wolf-Rayet star or two as having been overlooked in our survey, particularly in crowded regions, we believe this study underscores that there is not a significant number of W-Rs waiting to be discovered in the SMC. Thus W-R stars are under-abundant in the SMC by a factor of 3 (per unit luminosity) compared with the LMC. As we argue in Section 1, we believe that this supports the view that it requires a higher mass to become a $\mathrm{W}-\mathrm{R}$ in the SMC than it does in the LMC, due to the effect of lower metallicity on stellar winds.

Our discovery of an O5e If star, as well as three other hitherto unknown Of stars, emphasizes that much spectroscopic work remains to be done in the Magellanic Clouds. 
It is a pleasure to thank the CTIO support staff for their help. The Curtis Schmidt imaging kindly came about via director's discretionary time. A.S.D. participated in these studies partially through the Research Experiences for Undergraduates program, which was supported by the National science Foundation under grant 9988007 to Northern Arizona University. 


\section{REFERENCES}

Armandroff, T. E., \& Massey, P. 1985, ApJ, 291, 685

Azzopardi, M, \& Breysacher, J. 1979, A\&A, 75, 120

Azzopardi, M., \& Vigneau, J. 1975, A\&AS, 22, 285

Azzopardi, M., \& Vigneau, J. 1982, A\&AS, 50, 291

Barba, R. H., Niemela, V. S., Baume, G., \& Vazquez, R. A. 1995, ApJ, 446, 23

Belczynski, K., Mikolajewska, J., Munari, U., Ivison, R. J., \& Friedjunk, M. 2000, A\&AS, submitted (astroph/0005547)

Breysacher, J., Azzopardi, M., \& Testor, G. 1999, A\&AS, 137, 117

Breysacher, J., \& Westerlund, B. E. 1978, A\&A, 67

Conti, P. S. 1976, Mem. Soc. R. Sci. Liege, 6, Ser. 9, 193

Conti, P. S., \& Leep, E. M. 1974, ApJ, 193, 113

Conti, P. S. \& Massey, P. 1989, ApJ, 337, 251

Conti, P. S., Massey, P., \& Garmany, C. D. 1989, ApJ, 341, 113

Crowther, P. A., De Marco, O., \& Barlow, M. J. 1998, MNRAS, 296, 367

Garmany, C. D., Conti, P. S., \& Massey, P. 1987, AJ, 93, 1070

Hodge, P. 1985, PASP, 97, 530

Hodge, P. W., \& Wright, F. W. 1977, The Small Magellanic cloud (Seattle: University of Washington Press)

Hutchings, J. B., Crampton, D., Cowley, A. P., \& Thompson, I. B. 1984, PASP, 96, 811

Kennicutt, R. C. 1991, in The Magellanic Clouds, ed. R. Haynes \& D. Milne (Dordrecht, Kluwer), 139 
Kenyon, S. 1986, The Symbiotic Stars (Cambridge, Ambridge University Press)

Koenigsberger, G., Georgiev, L., Barba, R., Tzvetanov, Z., Walborn, N. R., Niemela, V. S., Morrell, N., \& Schulte-Ladbeck, R. 2000, ApJ, 542, 428

Lucke, P. \& Hodge, P. W. 1970, AJ, 75, 171

Maeder, A., \& Conti, P. S. 1994, ARA\&A, 32, 227

Massey, P. 1985, PASP, 97, 5

Massey, P. 2000, PASP, 112, 144

Massey, P., Armandroff, T. E., \& Conti, P. S. 1986, AJ, 92, 1303

Massey, P., DeGioia-Eastwood, K. \& Waterhouse, E. 2000a, AJ, submitted

Massey, P., \& Johnson, O. 1998, ApJ, 505, 793

Massey, P., Lang, C. C., DeGioia-Eastwood, K., \& Garmany, C. D. 1995, ApJ, 438, 188

Massey, P., Waterhouse, E., \& DeGioia-Eastwood, K. 2000b, AJ, 119, 2214

Meyssonnier, N., \& Azzopardi, M. 1993, A\&AS, 102, 451

Moffat, A. F. J. 1988, ApJ, 330, 766

Moffat, A. F. J., Breysacher J., \& Seggewiss, W. 1985, ApJ, 292, 511

Moffat, A. F. J., Niemela, V. S., \& Marraco, H. G. 1990, ApJ, 348, 232

Morgan, D. H., Vassiliadis, E., \& Dopita, M. A. 1991, MNRAS, 251, 51p

Morris, P. W., Eenens, P. R. J., Hanson, M. M., Conti, P. S., \& Blum, R. D. 1996, ApJ, 470,597

Neubig, M. M. S., \& Bruhweiler, F. D. 1997, AJ, 114, 1951

Niemela, V. S., Barba, R. H., \& Morrell, N. I. 1999, New Astron., 43, 475

Niemela, V. S., \& Morrell, N. I. 1999, in New Views of the Magellanic Clouds, ed. Y-H. Chu, N. B. Suntzeff, J. E. Hesser, \& D. A. Bohlender (San Francisco, ASP), 181 
Pyper, D. M. 1966, ApJ, 144, 13

Rodrigues, C. V., Magalhaes, A. M., Coyne, G. V., \& Piirola, S. J. V. 1997, ApJ, 485, 618

Russell, S. C., \& Dopita, M. A. 1990, ApJS, 74, 93

Shore, S. N., Sanduleak, N., \& Allen, D. A. 1987, A\&A, 176, 59

Smith, L. F. 1968a, MNRAS, 138, 109

Smith, L. F. 1968b, MNRAS, 140, 409

van den Bergh, S. 2000a, PASP, 112, 529

van den Bergh, S. 2000b, The Galaxies of the Local Group (Cambridge, Cambridge University Press)

van der Hucht, K. A., Conti, P. S., Lundstrom, I., \& Stenholm, B. 1981, Space Science Reviews, 28, 227

Walborn, N. R., \& Fitzpatrick, E. L. 1990, PASP, 102, 379

Westerlund, B. E. 1964, MNRAS, 127, 429

Zickgraf, F.-J., Wolf, B., Stahl, O., \& Humphreys, R. M. 1989, A\&A, 230, 206

Zubko, V. G. 1999, ApJ, 513, L29 
Fig. 1. - The region surveyed for Wolf-Rayet stars is shown by the 12 overlapping squares, each of which is $1.3^{\circ}$ on a side. The area surveyed by objective prism by Azzopardi \& Breysacher (1979) is shown by the 5 circles. The location of the $11 \mathrm{~W}-\mathrm{R}$ stars are indicated. The diagonal streak is an artifact on the Digitized Sky Survey image used to construct this diagram.

Fig. 2.- The normalized spectra of the 8 Azzopardi \& Breysacher (1979) W-R stars are shown, with the principle emission lines indicated. In (a) we show the weaker-lined stars, and in (b) we show the stronger-lined stars; the scaling is compressed a factor of 3 compared to that of $(\mathrm{a})$.

Fig. 3.- The normalized spectra of our two newly found SMC W-R stars (WR10 and WR11) are shown compared to the star found by Morgan et al. (1991), WR9. The scaling is the same as in $2(\mathrm{a})$.

Fig. 4.- Finding charts are presented for all 11 of the SMC W-Rs. Larger scale versions for SMC-WR1 through 8 appeared in Azzopardi \& Breysacher (1979), and for WR9 in Morgan et al. (1991). The ones shown here are 5 arcmin on a side, and were made from the Digitized Sky Survey; the circles marking the W-Rs are 20 arcsec in diameter. The following should be noted: WR4 is the brightest star in a small group. WR6 is slightly crowded; the correct star is the brighter star to the NW. WR7 has a faint companion to the NE. WR8 is the brightest star in the cluster NGC 602c, and a larger-scale chart can be found in Fig. 4 of Westerlund (1964); it is the star labeled 17.

Fig. 5.- Finding charts for the other interesting objects we found are given here. The finding charts are 5 arcmin on a side, and the circle marking the object is 20 arcsec in diameter.

Fig. 6.- The spectra of three of our newly found Of stars are shown; the fourth is shown 
in the following figure.

Fig. 7.- The spectrum of the newly found O5e If star is compared to the extreme O7 If+ star Sk 80. The latter spectrum was taken with our lower dispersion grating.

Fig. 8. - The spectrum of the $\mathrm{B}[\mathrm{e}]$ star S18 obtained in 2000 Oct is compared to that of S Dor when it was in its "low state" a 1996 Oct. The later spectrum comes from Massey (2000).

Fig. 9.- The spectrum of our newly found symbiotic star is shown. We show the spectrum preserving the flux scale (units are ergs $\mathrm{s}^{-1} \mathrm{~Hz}^{-1}$ ). The absolute flux has been adjusted by a factor of $1.5 \times$ to account for slit losses and bring the photometry into agreement with the CT magnitude. The spectrum closely resembles that of AG Dra; see Kenyon (1986), Fig. A14. The star was noticed as an $\mathrm{H} \alpha$ emission-line object by Meyssonnier \& Azzopardi (1993), who catalog it as number 1748. 
Table 1. Wolf-Rayet Stars in the SMC

\begin{tabular}{|c|c|c|c|c|c|c|c|c|c|c|c|c|}
\hline \multirow[t]{2}{*}{ Star } & \multirow[t]{2}{*}{ Other ID } & \multirow[t]{2}{*}{$\alpha_{2000}$} & \multirow[t]{2}{*}{$\delta_{2000}$} & \multirow[t]{2}{*}{$\mathrm{OB} / \mathrm{HII}^{\mathrm{a}}$} & \multirow[t]{2}{*}{ Spectral Type } & \multirow[t]{2}{*}{ Abs. } & \multirow[t]{2}{*}{$V$} & \multirow[t]{2}{*}{$B-V$} & \multirow[t]{2}{*}{$M_{V}$} & \multicolumn{2}{|c|}{$\mathrm{He}$ II or $\mathrm{C}$ III $^{\mathrm{b}}$} & \multirow[t]{2}{*}{ Comment } \\
\hline & & & & & & & & & & EW $(\AA)$ & FWHM $(A)$ & \\
\hline SMC-WR1 & AV $2 \mathrm{a}$ & 004342.23 & -732854.9 & no & WN3+abs & O3-4 & 15.14 & -0.04 & -4.6 & -28 & 21 & Weak abs. \\
\hline SMC-WR2 & AV 39a & 004830.81 & -731545.1 & near h15 & WN4.5+abs & O5: & 14.23 & -0.15 & -5.2 & -15 & 12 & \\
\hline SMC-WR3 & AV $60 \mathrm{a}$ & 004959.33 & -732213.6 & near h17 & WN3+abs & $\ldots$ & 14.48 & -0.10 & -5.1 & -53 & 26 & Very weak abs. \\
\hline SMC-WR4 & AV 81, Sk 41 & 005043.41 & -732705.1 & $\mathrm{~h} 21$ & WN6p & $\ldots$ & 13.35 & -0.16 & -6.2 & -45 & 15 & N V abs? \\
\hline SMC-WR5 & HD 5980 & 005926.60 & -720953.5 & NGC $346=\mathrm{h} 45$ & WN5 & $\ldots$ & 11.08 & +0.03 & -8.9 & -85 & 18 & \\
\hline SMC-WR6 & AV 332, Sk 108 & 010325.20 & -720643.6 & h53 & WN3+abs & O7 & 12.30 & -0.15 & -7.1 & -8 & 28 & \\
\hline SMC-WR7 & AV 336a & 010335.94 & -720321.5 & h53 & WN2+abs & O6 & 12.93 & -0.05 & -6.8 & -16 & 27 & \\
\hline SMC-WR8 & Sk 188 & 013104.22 & -732503.9 & NGC $602 c=h 69$ & WO4+abs & $\mathrm{O} 4 \mathrm{~V}$ & 12.81 & -0.14 & -6.6 & -76 & 71 & \\
\hline SMC-WR9 & Morgan et al. & 005432.17 & -724435.6 & no & WN3+abs & O3-4 & 15.23 & -0.13 & -4.3 & -22 & 24 & \\
\hline SMC-WR10 & & 004528.78 & -730445.2 & NGC 249(e12) & WN3+abs & O3-4 & 15.76: & $-0.08:$ & -3.6 & -24 & 23 & Strong neb. \\
\hline SMC-WR11 & & 005207.36 & -723537.4 & no & WN3+abs & O3-4 & 14.97 & +0.18 & -5.5 & -14 & 25 & \\
\hline
\end{tabular}

${ }^{a} \mathrm{OB}$ associations designation (h) are from Hodge (1985), and emission-line regions are from the Hodge \& Wright (1977) atlas.

${ }^{\mathrm{b}}$ The equivalent width (EW) and full-width-at-half-maximum (FWHM) of He II $\lambda 4686$ is given for the WNs; that of C III $\lambda 4650$ is given for the WO4 star. 
Table 2. Non W-R Stars of Interest

\begin{tabular}{llccccccl}
\hline \hline \multicolumn{1}{c}{ Star } & Other ID & $\alpha_{2000}$ & $\delta_{2000}$ & OB/HII ${ }^{\mathrm{a}}$ & $V$ & $B-V$ & $M_{V}$ & Spectral Type \\
\hline Of-type & & & & & & & & \\
MA93-344 & Lin 146 & 005011.30 & -732054.2 & no & 13.83 & $-0.2:$ & -5.4 & O9 If \\
Anon-1 & $\cdots$ & 005329.95 & -724144.2 & h35 & 13.98 & -0.18 & -5.3 & O5e If+ \\
Anon-2 & $\cdots$ & 005552.85 & -732235.3 & no & 13.41 & -0.15 & -6.0 & O7 If \\
AV398 & $\cdots$ & 010609.78 & -715600.3 & no & 13.88 & +0.09 & -6.2 & O8 If \\
Other & & & & & & & & \\
S18 & AV 154 & 005409.56 & -724143.2 & h35 & $13.7 \mathrm{var}$ & +0.3 & $-6.8:$ & B [e] \\
MA93-1748 & $\cdots$ & 011137.45 & -715901.8 & no & 16.61 & +1.04 & -2.6 & Symbiotic \\
\hline
\end{tabular}

${ }^{\mathrm{a}} \mathrm{OB}$ associations designation (h) are from Hodge (1985), and emission-line regions are from the Hodge \& Wright (1977) atlas. 
This figure "f1.jpg" is available in "jpg" format from: http://arxiv.org/ps/astro-ph/0010420v1 
This figure "f2a.jpg" is available in "jpg" format from: http://arxiv.org/ps/astro-ph/0010420v1 
This figure "f2b.jpg" is available in "jpg" format from: http://arxiv.org/ps/astro-ph/0010420v1 
This figure "f3.jpg" is available in "jpg" format from: http://arxiv.org/ps/astro-ph/0010420v1 
This figure "f4.jpg" is available in "jpg" format from: http://arxiv.org/ps/astro-ph/0010420v1 
This figure "f5.jpg" is available in "jpg" format from: http://arxiv.org/ps/astro-ph/0010420v1 
This figure "f6.jpg" is available in "jpg" format from: http://arxiv.org/ps/astro-ph/0010420v1 
This figure "f7.jpg" is available in "jpg" format from: http://arxiv.org/ps/astro-ph/0010420v1 
This figure "f8.jpg" is available in "jpg" format from: http://arxiv.org/ps/astro-ph/0010420v1 
This figure "f9.jpg" is available in "jpg" format from: http://arxiv.org/ps/astro-ph/0010420v1 